\title{
Efficient performance simulation of class $D$ amplifier output stages
}

\author{
Nyboe, Flemming; Risbo, Lars; Andreani, Pietro
}

Published in:

Norchip 2005

Link to article, DOI:

10.1109/NORCHP.2005.1596982

Publication date:

2005

Document Version

Publisher's PDF, also known as Version of record

Link back to DTU Orbit

Citation (APA):

Nyboe, F., Risbo, L., \& Andreani, P. (2005). Efficient performance simulation of class D amplifier output stages. In Norchip 2005 (pp. 32-35). IEEE. https://doi.org/10.1109/NORCHP.2005.1596982

\section{General rights}

Copyright and moral rights for the publications made accessible in the public portal are retained by the authors and/or other copyright owners and it is a condition of accessing publications that users recognise and abide by the legal requirements associated with these rights.

- Users may download and print one copy of any publication from the public portal for the purpose of private study or research.

- You may not further distribute the material or use it for any profit-making activity or commercial gain

- You may freely distribute the URL identifying the publication in the public portal

If you believe that this document breaches copyright please contact us providing details, and we will remove access to the work immediately and investigate your claim. 


\title{
Efficient Performance Simulation of Class D Amplifier Output Stages
}

\author{
FLEMMING NYBOE ${ }^{1}$, LARS RISBO ${ }^{2}$ AND PIETRO ANDREANI ${ }^{3}$ \\ ${ }^{1}$ Digital Audio Department, Texas Instruments, Denmark \\ and \\ Orsted $\cdot D T U$, Technical University of Denmark \\ fls (a)ti.com \\ ${ }^{2}$ Digital Audio Department, Texas Instruments, Denmark \\ Iri@ti.com \\ ${ }^{3}$ Orsted $\cdot$ DTU, Technical University of Denmark \\ pa $a$ oersted.dtu.dk
}

\begin{abstract}
:
Straightforward simulation of amplifier distortion involves transient simulation of operation on a sine wave input signal, and a subsequent FFT of the output voltage. This approach is very slow on Class $D$ amplifiers, since the switching behavior forces simulation time steps that are many orders of magnitude smaller than the duration of one period of an audio sine wave. This work presents a method of simulating the amplifier transfer characteristic using a minimum amount of simulation time, and then deriving THD from the results.
\end{abstract}

\section{Introduction}

A simplified schematic of a class D audio amplifier system is shown in Figure 1. The audio input signal (analog or digital) is converted to a logic-level pulse width modulated (PWM) signal by a modulator (not shown), and level shifted to produce the gate signals for the switches. The two output stage switches are turned on alternately, reproducing the PWM waveform at the switching node $\mathrm{V}_{\mathrm{SW}}$. The demodulation LC filter then removes the switching frequency components of the PWM signal, leaving only the audio signal on the output node $\mathrm{V}_{\text {out }}$.

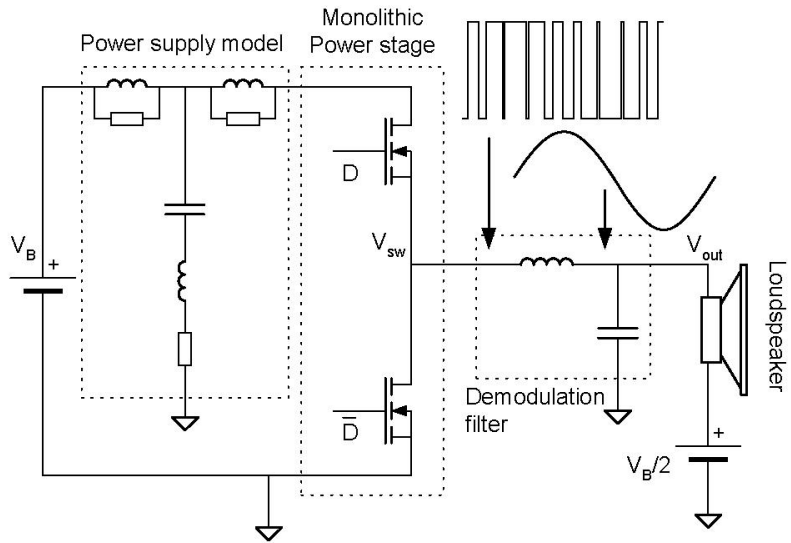

Figure 1: Simple Class D amplifier system. The input signal is the duty cycle (D) of the gate signals for the switches.
Typical switching frequencies are in the range of $300-$ $800 \mathrm{kHz}$. One period of the PWM signal is referred to as a frame. For the case of switching duty cycle $D=0.5$, the output voltage $V_{\text {out }}$ equals $V_{B} / 2$, leaving zero voltage across the loudspeaker. This case is referred to as idle operation.

One of the key performance metrics for audio amplifiers is THD vs. output signal amplitude. Straightforward simulation of THD for the system shown in Figure 1 would involve a transient simulation where the duty cycle D was varied in a purely sinusoidal fashion around $\mathrm{D}=0.5$, at an audio frequency, e.g. $5 \mathrm{kHz}$. THD could then be found from an FFT of the simulated voltage $V_{\text {out }}$. The problem about this approach is runtime. The switching transitions force the use of small time steps in simulation, and since the switching frequency is about two orders of magnitude higher than $5 \mathrm{kHz}$, many PWM frames must be simulated to reproduce a single period of a sine wave audio signal. While similar simulation challenges have been treated in literature, mainly within $\mathrm{RF}$ electronics [1], this work focuses on switching power amplifiers.

Using a higher frequency audio signal obviously shortens the needed transient simulation time, but as audio signal frequency is increased, THD contributions will disappear gradually from the simulation result for two reasons: Firstly, the output LC filter will filter out the higher order harmonic components. This problem is easily avoided by deriving THD from an FFT of $\mathrm{V}_{\mathrm{SW}}$ instead of $\mathrm{V}_{\text {out, }}$, and then applying a filter with higher cutoff frequency in computation, thereby ignoring frequency components associated with the PWM switching. Secondly, too small a ratio between the switching frequency and the audio frequency corresponds to a coarse sampling of the nonlinearities present in the output stage, hiding some THD contributions from the result, and there is no similar workaround for this problem. For example, to accurately simulate distortion originating from switching dead time, many closely spaced values of PWM duty cycle in the vicinity of $\mathrm{D}=0.5$ are needed [2]

\section{Simulation of the Transfer Characteristic}

An alternative to the straightforward performance simulation approach described above is to simulate the 
input/output transfer characteristic of the amplifier once, and then derive THD vs. signal amplitude from the result. This assumes that the transfer characteristic from duty cycle $\mathrm{D}$ to output voltage $\mathrm{V}_{\text {out }}$ is indeed constant, i.e. not dependant on audio signal frequency. This assumption is valid for the nonlinearities in an open loop Class D output stage, since the switching waveforms depend only on the current in the LC filter inductor at the time of switching.

In practice, Class D amplifier distortion can depend significantly on audio signal frequency. This is partly due to the frequency dependency of the $V_{B}$ power supply output impedance, and partly to temperature in the output stage varying at the audio frequency. The first effect is not related to the output stage itself, and considered outside the scope of this paper, and the second is not seen in simulation, regardless of approach, as long as the simulator does not incorporate device self heating. Finally, analog-input Class D amplifiers typically use feedback, which will cause distortion to increase with frequency as the feedback loop looses gain. However, amplifiers with feedback can not easily be simulated using the principles described here anyway, since the PSS shooting algorithm (mentioned below) is not likely to converge. If the feedback loop is opened, the simulation approach described here can be applied to the feed-forward path of the amplifier, and still be a useful tool to assess linearity.

For convenience, the amplifier transfer characteristic TC is defined as:

$V N_{\text {out }}=T C(D N)$

where (referring to Figure 1)

$$
V N_{\text {out }}=\frac{2 \cdot V_{\text {out }}}{V_{B}}-1 \text { and } D N=2 \cdot(D-0.5)
$$

This means that both $\mathrm{DN}$ and $\mathrm{VN}_{\text {out }}$ will be normalized to the range $-1 . .1$, and for an ideal distortion free amplifier, TC would be linear with slope 1 , i.e. $\mathrm{VN}_{\text {out }}=$ DN. Once this normalized transfer characteristic has been determined, the output signal at any amplitude can be calculated (rather than simulated) using the following equation:

$$
V N_{\text {out }}(t)=T C\left(10^{\frac{A}{20}} \cdot M \cdot \sin (t)\right) \quad \mathrm{t}=0 . .2 \pi
$$

and THD vs. signal amplitude can be found from a subsequent FFT of the result. Here $A$ is the audio signal amplitude of interest (in $\mathrm{dB}$ ), and $\mathrm{M}$ is the maximum modulation index. $M$ is typically in the range 0.95 to 0.98 and accounts for the fact that most Class D output stages have a lower bound on PWM pulse width, meaning that the actual duty cycle range at $A=0 \mathrm{~dB}$ is slightly narrower than 0 to 1 . Note that for each signal amplitude of interest, THD can be found using equation (2) and an FFT calculation, no extra simulation is needed like the straightforward simulation approach described in the introduction.

\section{Selection of $D$ values and Transfer Characteristic Interpolation}

In order to determine TC(DN) for a system, a number of duty cycle values $\mathrm{DN}$ must be selected, and $\mathrm{VN}_{\text {out }}$ simulated for each value. In order to use equation (2), TC(DN) must be defined for any DN in the range $-1 . .1$, and since it can only be simulated for a finite number of input values DN, interpolation is needed. Figure 2 shows an example of a transfer characteristic that is simulated for 7 values of $\mathrm{DN}$, and then reconstructed using linear or cubic spline interpolation.

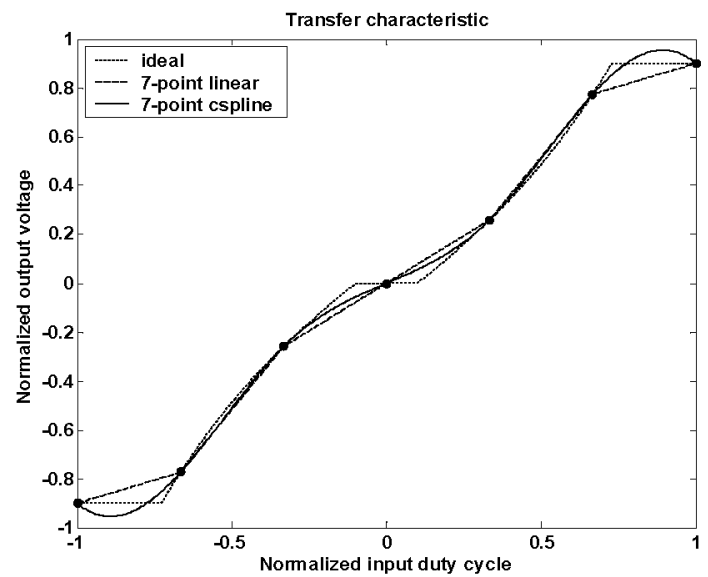

Figure 2: Amplifier transfer characteristic VN=TC(DN), simulated for 7 linearly spaced duty cycle values and recontructed using linear or cubic spline interpolation.

The ideal transfer characteristic shown in Figure 2 is given by

$$
T C(D N)= \begin{cases}V N_{\text {out }}=d 3 \cdot D N^{3} & D N \leq z c \\ V N_{\text {out }}=D N+d 3 \cdot D N^{3} & D N>z c\end{cases}
$$

$V N_{\text {out }}$ limited to $\pm V N_{\text {clip }}$

where $\mathrm{d} 3$ is the $3^{\text {rd }}$ order distortion coefficient, $\mathrm{zc}$ is the bound for zero-crossing distortion, where gain is reduced for $|\mathrm{DN}|<\mathrm{zc}$. $\mathrm{VN}_{\text {clip }}$ is the output clipping limit.

These properties represent common amplifier nonlinearities ${ }^{1}$. For the curves in Figure 2, d3 $=0.7$, $\mathrm{zc}=0.1$ and $\mathrm{VN}_{\mathrm{clip}}=0.9$. The degree of nonlinearity is vastly exaggerated in order to aid visibility in Figure 2. For any reasonable audio amplifier, the actual nonlinearity would not be visible in such a plot.

In the example shown, the $7 \mathrm{DN}$ values at which $\mathrm{TC}(\mathrm{DN})$ is simulated are linearly spaced. This poses a problem when equation (2) is used to derive THD at

\footnotetext{
${ }^{1}$ Though Class D amplifiers do not exhibit zero crossing distortion in a traditional sense, they do have a nonlinearity associated with switching dead time that causes reduced gain at low signal amplitudes. See [2] for a detailed discussion.
} 
small signal amplitudes from the interpolated TC(DN) function. E.g. if THD at $\mathrm{A} \leq-10 \mathrm{~dB}$ is derived from one of the interpolated transfer characteristics in Figure 2, the result will be based almost exclusively on the 3 points at and around $\mathrm{DN}=0$ where $\mathrm{TC}(\mathrm{DN})$ has been simulated ${ }^{2}$. Obviously this problem is reduced when more points are used, but even for $201 \mathrm{DN}$ value points a similar problem will occur for $A \leq-40 \mathrm{~dB}$.

Improved resolution at small signal amplitude can be achieved by distributing the DN values unevenly, with a higher concentration in the vicinity of $\mathrm{DN}=0$. Instead of a linear spacing, we propose distributing $\mathrm{m}$ points as follows:

$$
D N=0, \pm \log _{10}\left(\frac{2 k}{m-3} \cdot \frac{\text { lvlmin }}{20}\right) \quad k=0 . . \frac{\mathrm{m}-3}{2}
$$

where $m$ must be odd

There is a data point at $\mathrm{DN}=0$, and logarithmically distributed values from lvlmin $\mathrm{dB}$ towards $0 \mathrm{~dB}$ in both the positive and negative direction. For example, for $\mathrm{m}=$ 7 and $l$ lmin $=-40 \mathrm{~dB}$ we get

$$
D N=(-1,-0.1,-0.01,0,0.01,0.1,1)
$$

Since this distribution is logarithmic in nature, but includes zero and both positive and negative values, we will refer to it as pseudo-logarithmic here.

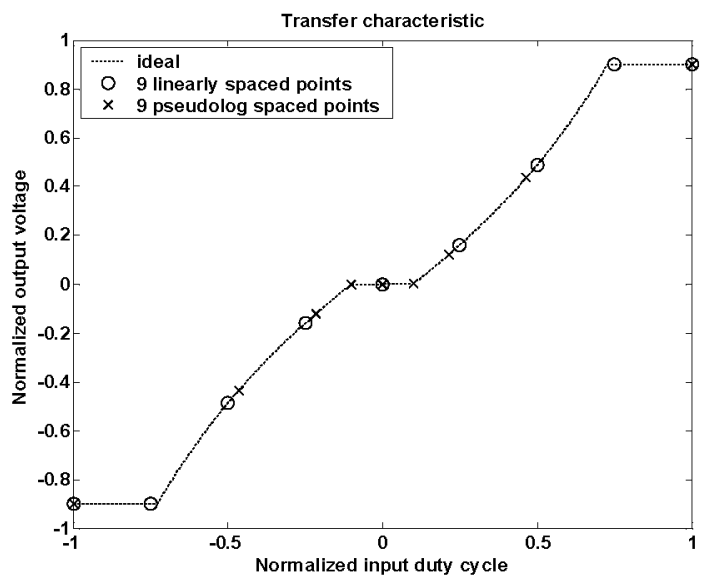

Figu re 3: TC(DN) simulated at 9 DN values, with linear versus pseudo-logarithmic distribution

For a given number of DN points, the pseudologarithmic distribution greatly increases the accuracy of THD calculations for small signal amplitudes, at the penalty of a decrease in accuracy at large signal amplitudes.

\section{THD matching}

To test the accuracy of THD simulation using different algorithms for DN value spacing and transfer

\footnotetext{
${ }^{2}$ When using cubic spline interpolation, the interpolated TC(DN) function does depend sligthly on data points further from DN.
}

characteristic interpolation, THD for the nonlinearity described by equation (3) is used as a test case. Rather than using a measured transfer characteristic TC(DN) from an actual Class D amplifier, this has the advantage that due to the symbolic nature of the equation, there is a definite correct answer to the THD vs. amplitude curve. For this purpose, the parameters for equation (3) are set at values that realistically represent an audio amplifier: $\mathrm{d} 3=0.003, \mathrm{zc}=5 \cdot 10^{-5}, \mathrm{VN}_{\text {clip }}=0.9$

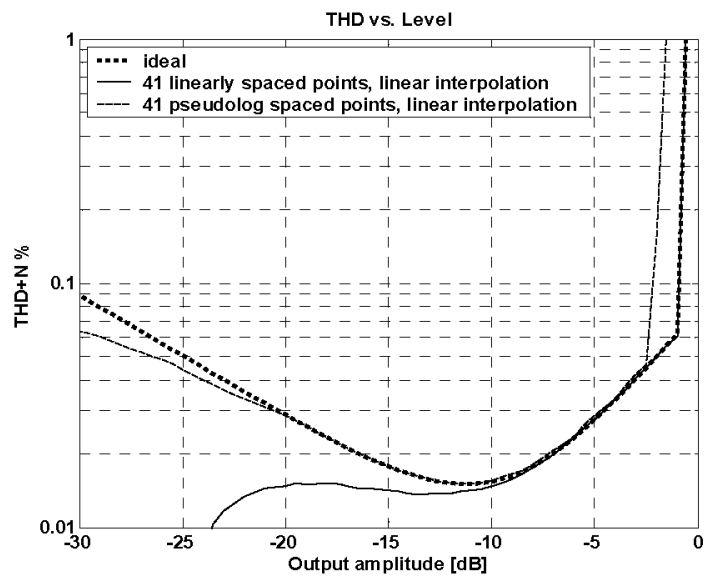

Figure 4: THD reconstruction using 41 simulation points and linear interpolation between them. THD based on all harmonics from an FFT of size 64, M=1 (refer to equation 2).

Figure 4 shows THD calculation results based on 41 simulation points, spaced linearly and pseudologarithmically. Linear interpolation between the data points is used in both cases. As expected, the linear distribution results in too small THD numbers at small signal amplitude, because the density of simulated data points $\mathrm{TC}(\mathrm{DN})$ is too low to model the zero-crossing distortion. Conversely, the pseudo-logarithmic distribution models the clipping at large signal amplitude inaccurately.

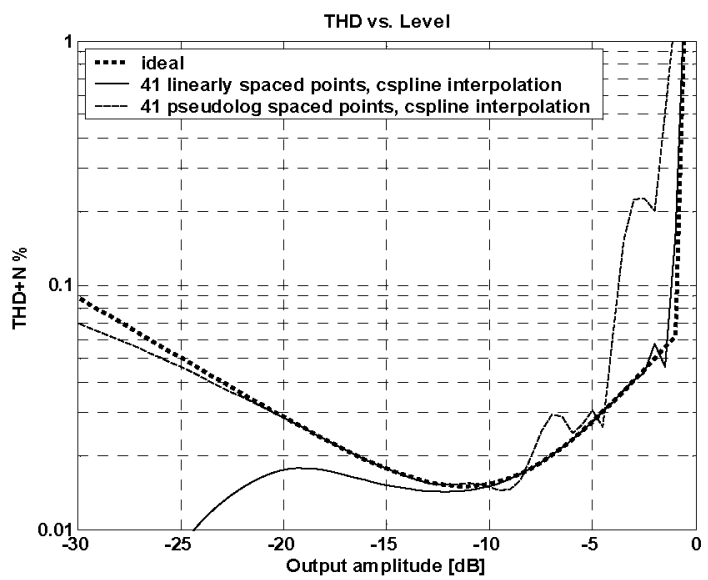

Figure 5: THD reconstruction using 41 simulation points and cubic spline interpolation between them. THD based on all harmonics from an FFT of size 64, $\mathrm{M}=1$ (refer to equation 2).

The clipping sets in too early, because the density of data points is low, and the linear interpolation results in a less 
aggressive clipping between the data points at $\mathrm{DN}= \pm 1$ and their nearest neighbors, which are below the actual clipping level. This phenomenon can also be observed in Figure 2 (linear interpolation).

Figure 5 shows the same THD calculation, except cubic spline interpolation is used between the simulated data points. While cubic spline interpolation may generally be conceived as superior to linear interpolation, it is not the case for this purpose. For small signal amplitudes, the results are about equal to those from linear interpolation, but the cubic spline causes large inaccuracies in the modeling of clipping, especially in combination with the pseudo-logarithmic distribution of data points, again because of its coarse resolution of $\mathrm{DN}$ values at large signal amplitudes. This is not surprising, since ideal clipping as described by equation (3) is an abrupt kink in the transfer characteristic, and cubic splines are free of kinks by design.

Cubic spline interpolation may still provide superior accuracy on actual designs that do not have sharp kinks in their transfer characteristic. This can be tested by running one (CPU intensive) simulation with a very large number of data points, and then test which interpolation method that best matches the high-resolution THD result with fewer data points. When a useful resolution, spacing algorithm and interpolation method has been found, it can be used in design optimization, and the final solution verified with another high-resolution simulation.

\section{Computational efficiency}

For comparison to a straightforward transient simulation of a period of an audio sine wave on $\mathrm{V}_{\text {out }}$, consider the following: One sine wave period at $5 \mathrm{kHz}$ audio frequency would require 200us transient simulation, plus additional time for the output LC filter to stabilize before the FFT window starts. Assuming another 100us is needed for stabilization, this is $300 \mathrm{us}$ of transient simulation per THD vs. amplitude data point. Note that even if THD is derived from $V_{S W}$ rather than $V_{\text {out }}$, the output filter influences linearity by generating switching ripple current, which in turn influences the switching transition waveforms and thus THD. This means that the LC output filter cannot be disregarded in simulation, and it must settle in order to find the periodical output sine wave. Computing the initial conditions for the LC filter components at the start of each transient simulation, with as good accuracy as possible, is obviously crucial for fast settling.

Assuming that a THD vs. amplitude plot is to be generated, for $-40 \mathrm{~dB}$ to $0 \mathrm{~dB}$ signal amplitude in $1 \mathrm{~dB}$ steps, a total of $12.3 \mathrm{~ms}$ transient simulation time is needed. If 41 CPUs are available, the wall clock time is that of simulating $300 \mathrm{us}$.

Using the transfer characteristic approach, the accuracy seen in Figure 4 and Figure 5 can be achieved with 41 data points. For a Class $\mathrm{AB}$ amplifier, each data point would be just an operating point simulation, but for a Class D amplifier, it requires a Periodic Steady State (PSS) simulation to find the output voltage Vout (equal to the average of VSW) for a given duty cycle DN. For a description of PSS, refer to [3]. The PSS shooting algorithm typically takes 5 iterations to find the steady state, so at $400 \mathrm{kHz}$ PWM switching frequency that means 12.5 us transient simulation time per data point. This is 512 us total transient simulation time, or just about $4 \%$ of the $12.3 \mathrm{~ms}$ needed for transient simulations of the audio signal.

While the transfer characteristic approach has inaccuracy associated with DN value spacing algorithms, simulation of audio sine wave operation has a very similar inaccuracy related to the choice of audio frequency. Reproducing an audio sine wave with a fixed PWM frequency corresponds to sampling the transfer characteristic at a finite number of points spaced linearly in time. E.g. playing back one period of a $5 \mathrm{kHz}$ audio signal at $400 \mathrm{kHz}$ switching frequency provides 80 data points. Because of the repetitive nature of a sine wave, half of these points are repetitions of points already simulated, and do not contribute any additional accuracy. The data set thus contains 40 unique data points, and the sine wave shape causes a high density of data points at large duty cycle values and a low density around idle. While the straightforward approach has the advantage that these 40 data points are always within the range of the audio signal, regardless of amplitude, the lower resolution around idle is likely to impact accuracy of e.g. the THD contribution related to dead time at medium signal amplitudes.

\section{Summary}

An indirect performance simulation method for Class D amplifiers has been presented. Like most simulations, it possesses basic trade offs between CPU time and accuracy, but it is easily more efficient than transient simulation of sine wave amplification. The method is based on the assumption that the transfer characteristic of the amplifier is time invariant. This is true for open-loop Class D amplifier output stages if distortion related to finite PSU impedance and temperature modulation is disregarded. For amplifiers with feedback, the approach can only be applied with the feedback loop opened, but this can still be useful for assessing linearity of the feedforward path.

\section{References}

[1] Efficient Methods for Simulating Highly Nonlinear Multi-Rate Circuits

Jaijeet Roychowdhury

Bell Laboratories, Murray Hill, USA

1997 Design Automation Conference, Anaheim, CA

[2] Time Domain Analysis of Open Loop Distortion in Class D Amplifier Output Stages

Flemming Nyboe, Lars Risbo, Pietro Andreani $27^{\text {th }}$ International AES Conference. September 2005.

[3] Virtuoso@ SpectreRF Simulation Option User Guide "Periodic Steady-State Analysis" chapter. http://analog.ee.washington.edu/pdf/spectreRF.pdf (7419KB) 